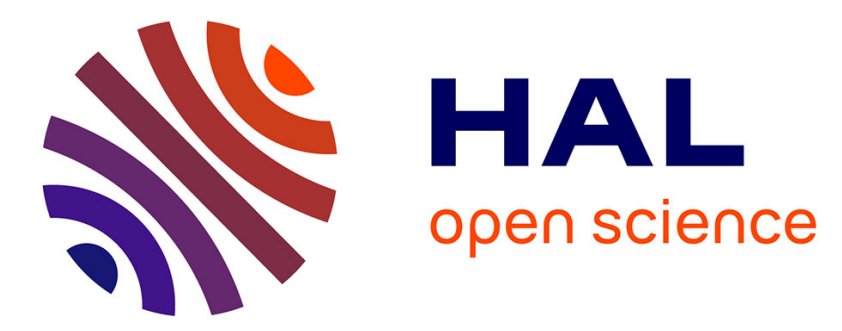

\title{
Real-time light dosimetry for intra-cavity photodynamic therapy: Application for pleural mesothelioma treatment
}

Nacim Betrouni, Camille Munck, Wael Bensoltana, Grégory Baert, Anne-Sophie Dewalle-Vignion, Arnaud Scherpereel, Serge Mordon

\section{- To cite this version:}

Nacim Betrouni, Camille Munck, Wael Bensoltana, Grégory Baert, Anne-Sophie Dewalle-Vignion, et al.. Real-time light dosimetry for intra-cavity photodynamic therapy: Application for pleural mesothelioma treatment. Photodiagnosis and Photodynamic Therapy, 2017, Epub ahead of print. 10.1016/j.pdpdt.2017.02.011 . inserm-01484376

\section{HAL Id: inserm-01484376 https://www.hal.inserm.fr/inserm-01484376}

Submitted on 7 Mar 2017

HAL is a multi-disciplinary open access archive for the deposit and dissemination of scientific research documents, whether they are published or not. The documents may come from teaching and research institutions in France or abroad, or from public or private research centers.
L'archive ouverte pluridisciplinaire HAL, est destinée au dépôt et à la diffusion de documents scientifiques de niveau recherche, publiés ou non, émanant des établissements d'enseignement et de recherche français ou étrangers, des laboratoires publics ou privés. 


\section{Real-time light dosimetry for intra-cavity photodynamic therapy: Application for pleural mesothelioma treatment}

Nacim Betrouni ${ }^{1,2}$, Camille Munck ${ }^{1,2}$, Wael Bensoltana ${ }^{1}$, Grégory Baert ${ }^{1}$, Anne-

Sophie Dewalle-Vignion ${ }^{1}$, Arnaud Scherpereel ${ }^{2}$, Serge Mordon ${ }^{1,2}$

${ }^{1}$ INSERM, U1189, 1, Avenue Oscar Lambret, 59037, Lille Cedex, France

${ }^{2}$ Lille University Hospital -59037 Lille Cedex, France

\section{Corresponding author}

Nacim Betrouni

INSERM, U1189 ONCO-THAI

1, Avenue Oscar Lambret

59037 Lille Cedex

France

Tel: 33. 3. 20.44.67.22

email: nacim.betrouni@inserm.fr 
Abstract. Complete and homogeneous illumination of the target is necessary for the success of a photodynamic therapy (PDT) procedure. In most applications, light dosimetry is done using detectors placed at strategic locations of the target. In this study we propose a novel approach based on the combination of light distribution modeling with spatial localization of the light applicator for real time estimation and display of the applied dose on medical images. The feasibility approach is demonstrated for intrapleural PDT of malignant pleural mesothelioma.

Keywords: photodynamic therapy, intra-cavity application, light distribution modeling, dosimetry, real time tracking, imaging. 


\section{1- Introduction}

Photodynamic therapy (PDT) is an emerging technic in oncology. Its mechanism of action is based on the interaction of a photosensitizer (PS) drug, a light source for the PS activation and oxygen in tissues. The combination of these three elements induces chemical reactions leading to the creation of reactive oxygen species (ROS) and tumor cell death.

The achievement of the desired therapeutic effect of a PDT procedure relies on the accurate PS concentration distribution and on the continuous and uniform light delivery to the tissues. This last issue is particularly critical due to the variability in target geometry: solid, hollow organs and cavities,...

For cavities, various types of light applicators were investigated as bare-fibers, having a directed radiation pattern and being applicable for small lesions, cylindrical diffusers [1] with scattering dome applicators which has a homogeneously distributed radiation pattern with various active lengths and lastly, flat or flexible 2D applicators as textile [2] or blanket [3] allowing obtaining planar illumination.

In addition to these applicators, sensors are used to monitor light application by the continuous measurement of the light fluence rate in different locations in the cavity. Most often, these sensors consist of isotropic probes collecting light from every direction [4]. This dosimetry method allows only obtaining punctual measurements of light distribution whereas continuous spatial feedback is required to estimate received dose at each target point.

This is particularly the case for Malignant Pleural Mesothelioma (MPM) treatment. Treating MPM remains a challenge with two main alternatives: palliative chemotherapy for inoperable patients, and multimodal treatment including surgery, combined with chemotherapy and radiotherapy for the others.

Surgery offers the best chance of survival for this still incurable disease, however after the most complete tumor resection, microscopic tumor cells persist and surgery should be associated with an adjuvant local treatment. PDT appeared as a potential option for an effective intra-operative complement to surgery. After extensive preclinical studies, first clinical results were reported by the University of Bern group [5]. Currently, the most complete studies, with a major impact on survival and minimum toxicity, are certainly those led by the Pennsylvania team [6-8]. The 
photosensitizer (Porfimer sodium (Photofrin)) is administered to the patients 48 hours before a maximal surgical tumor resection. Then, a light applicator connected to a laser source (wavelength $635 \mathrm{~nm}$ ) is moved inside the pleural cavity, to illuminate the cavity walls (the target), filled with dilute intra-lipid solution, until a fluence of $20 \mathrm{J.cm}$ -

${ }^{2}$ is obtained. The light delivery monitoring is achieved by 7 isotropic probes placed at strategic locations in the thorax and connected to a dosimetry system ([9], [4;10]).

However, this method does not provide information about the light delivered between these 7 locations. In this study, we describe a light applicator and introduce a new method for light dosimetry during PDT procedures of intra-cavities with complete and continuous feedback of the applied light dose. We present the feasibility of this approach on a phantom mimicking an intraoperative thorax cavity.

\section{2- Materiel and Methods}

The global approach proposed herein is based on three ideas. First, we present an unique action model that characterizes the light distribution around the applicator. The second idea is about using a spatial locator for real time tracking of the applicator movements inside the cavity in order to cumulate spatially and temporally the light dose applied to the target. The last idea requires the use of imaging to define the target and to be used as a spatial reference map for the real time display of the applied light dose.

\section{2-1 Light applicator construction and setting}

The proposed light applicator is an adaptation of the model used in the previous trials [11]. It consists of a cylindrical diffuser held in an endo-tracheal tube in which a carbon tube was inserted, to harden the tube and to correct its curvature. The cuff of the endo-tracheal tube is filled with intra-lipid solution at the concentration of $0.01 \%$ to act as a scattering agent.

For the real time localization of the applicator, an electromagnetic 3D tracking device is used to track the applicator movements. This tracking system (TrackStar, Ascension Technology Corporation, Burlington, VT, USA) is composed of a control unit with plugs to connect a transmitter and up to 4 six degrees-of-freedom sensors. One sensor ( $2 \mathrm{~mm}$ diameter at the distal end and $1.2 \mathrm{~mm}$ diameter for the cable) is fixed just above the cylindrical diffuser (Figure 1.a). It allows the localization of the sensor, and therefore the light wand, giving a 3D position and orientations regarding 
to the reference defined by the transmitter with a precision of $0.5 \mathrm{~mm}$. Figure 1.b represents the light wand assembled.

\section{2-2 Characterization of a light distribution model}

Light distribution around the diffusing tip of the applicator was characterized by direct measurements of the light fluence rate (irradiance $\left(\mathrm{W} . \mathrm{cm}^{-2}\right)$ ) using sensors completed with a digital photography to define fluence rate isosurfaces.

Measurements were done in a plastic tank of black color, limiting light reflection, filled with $0.01 \%$ dilute intralipid. The tip of the applicator was fixed horizontally in the tank through a cable gland and the optical fiber was connected to a medical diode laser 635 (Ceralas $®$, Biolitec)..

For the fluence rate measurements, an isotropic probe (Model IP 85, Medlight ${ } S . A$., Switzerland), collecting light in a large solid angle, was connected to an optical power meter (Model 841-PE,Newport ${ }^{\circledR}$ Corporation, Irvine, CA, USA). The isotropic probe was fixed vertically in a plastic tube and moved above the diffusing tip of the light wand, using a millimetric precision benchmark with vertical and horizontal freedom degrees.

A calibration factor was defined to convert the punctual power measurements (W) into fluence rate values $\left(\mathrm{W} . \mathrm{cm}^{-2}\right)$. It was estimated by correlating pairwise power measures obtained by the isotropic probe and fluence measures obtained by a $1 \mathrm{~cm}^{2}$ surface detector (Model PD300, OPHIR, Israel).

The previous method allowed only obtaining measurements for discrete positions on the light applicator. This dosimetry method with the powermeter only gave punctual measurements of the light distribution. In order to obtain a more complete and continuous light distribution characterization, a digital photography was taken above the tank. After spatial and chromatic calibrations of the picture, the measurements done previously and their respective positions were matched with the image to define iso-surfaces as represented in figure 2.

Complete description of the method is provided in Munck et al. [12].

\section{2-3 Tridimensional Imaging}

Radiation therapies (radiotherapy and brachytherapy) are always image-based techniques. Imaging allows to define the targets, to estimate their volumes and in 
some cases, to define organs at risk to be spared. It is also used to optimize the ballistics and as a reference map for the dose display.

Regarding PDT, imaging is being increasingly used especially for interstitial applications as in prostate [13], Glioblastoma [14] and head and neck [15;16] diseases. As for radiotherapy, the goal is mainly to define the targets to treat.

For MPM management, 3D computed tomography (CT) imaging is the reference for pleural evaluation, fortumor staging and follow-up. We propose to use these images in the dosimetry process of the PDT procedure. First, these images are used to estimate volume and surface of the pleural cavity. Thereby, the total required fluence and light application duration can be estimated before the treatment.

Another benefit to CT scan images is to use them as a reference space for the real time light dose display during the treatment. However, as these images are acquired before the procedure, a spatial registration is required to compute the transformation between the pre-operative space and the per-operative space. Fiducial markers consisting of $5 \mathrm{~mm}$ diameter capsule filled with paraffin oil can be attached to the patient around the region to be imaged. These markers are multimodality imaging compatible and able to create clear patterns on the images.

\subsection{Spatial mapping of light dose}

In order to compute a 3D light dose (D), the action model established for the applicator is combined with the positions returned by the spatial locator. The dose is matched with the images space using the following transformation:

$$
T_{\text {Applicator }}^{\text {images }}=T_{\text {Transmitter }}^{\text {images }} \circ T_{\text {Applicator }}^{\text {Transmitter }}
$$

$T_{\text {Applicator }}^{\text {Transmitter }}$ is formed using the positions and the orientations given by the tracking device and continuously updated according to the tracking frequency rate, while $T_{\text {Transmitter }}^{\text {images }}$ is the registration transformation that locates the patient regarding to the localization system. It is computed by mapping the coordinates of the fiducial markers on the images and their coordinates in the tracking device space (transmitter space). These last coordinates are obtained using a second sensor that points them successively.

Once the $3 D$ spatial mapping done, for each voxel point $p(x, y, z)$, the cumulated 
dose is estimated as:

$$
D_{p(x, y, z)}=\int_{\text {Treatment time }} \operatorname{IrradianceModel}(x, y, z) \cdot d t
$$

Where IrradianceModel is the above defined unique action model that characterizes the light applicator.

\section{3- Validation of the approach}

\section{3-1 Protocol}

Experiments are done on an anatomically realistic phantom consisting of a humansized hemithorax, made of plaster, and 3D printed into a right-side intra-operative and post tumor resection pleural cavity (Figure 3).

Four fiducial markers were attached to the phantom and CT images were acquired using standard protocol used for MPM exam (Figure 4).

A PDT procedure is simulated by moving the light applicator inside the cavity. The evaluation protocol consists in comparing the light dose measured by our proposed method and by the method of reference wi the 7 isotropic probes. First, the 3D positions of the probes are obtained using a spatial locator for each position; the model estimated fluence is extracted from the matrix $D$ (equation 2). These values are referred as Model_Measurements. The values collected by the probes are referred as Ref_Measurements.

Six independent experiments (Exp1 to Exp6) were carried out by varying the light applicator movements (speed, trajectory,...) inside the cavity.

\section{3-2 Results}

Figure 5 shows the accumulated 3D light distribution (matrix D) combined with the CT images to locate the treated and untreated regions. A special color look up table (LUT) gives relative display regarding a reference dose value. Each color represents $10 \%$ of this dose.

Figures 6 depicts the temporal evolution of the cumulated fluence $\left(\mathrm{J} . \mathrm{cm}^{-2}\right)$ measured by the two methods in one of the 7 intrathoracic locations (probes) respectively for (Exp1, Exp2, Exp5), and (Exp3, Exp4, Exp6). From this figure, it appears that the Ref_Measurements have a linear evolution. This result can be explained by the fact 
that the probes were continuously collecting light through the intralipid liquid even when the applicator was far from their positions, while the Model_Measurements do not have this ability because the model has a limited volume as defined in section 2.2 . For the 7 probes, the mean error between the reference measurements and the estimated measurements was about $10 \%$ with under-estimation for the proposed method. Since this under-estimation was constant for the six experiments, a correction factor of 1.1 was established and a new experiment was carried out after correcting the Model_Measurements with this value. Figure 7 presents the temporal evolution of the mean light dose calculated in one position for all experiments after correction.

The difference between the two methods measurements decreased below $1 \%$.

\section{4- Discussion}

The success of a PDT procedure depends on the optimal dosimetric configurations of the photosensitizer and the light. The first issue relies on the determination of the PS distribution to the target tissues, which remains particularly difficult to monitor in clinical conditions. In most applications, based on dose escalation trials, a defined concentration is considered as optimal with sufficient and homogeneous distribution in tissues, and limited toxicity. For Photofrin, used in PDT for MPM, a dose of 2mg/ $\mathrm{Kg}$ is commonly administered to the patient.

The second issue, light delivery, is to apply a sufficient light dose to each target point. The approach proposed herein constitutes a new trend in dosimetry for intrapleural PDT. Indeed, instead of the continuous measurement of the light delivered, using dedicated materiel (probes, spectrometer, power-meter,...), our dosimetry method relies on a unique action model that characterizes the light propagation from the light applicator. The model was established by defining the light distribution profile in the intra-lipid solution. It takes into account the absorption and the diffusion around the applicator but not the multiple scattering that occurs within the intralipid solution. This can explain the systematic under-estimation of the model.

Anyway, in this kind of treatment, the procedure is based on a continuous movement of the light source inside the cavity, which means that the time spent at a specific spatial position is short leading to a non significant impact of the light scattering. 
The second originality of our dosimetry method resides in the real time tracking of light applicator's movements inside the cavity, using a spatial locator. Thus, by combining the model and the positions, a complete 3D light dose is estimated. Spatial locators were already used in laser applications to track the light applicator movements ([17], [18]) and were proved to be efficient tools to monitor light application, in addition to being safe and clinically suitable.

Actually, from a clinical point of view, the real time update and display of the applied light dose brings the physician three main benefits. The first is the continuous feedback about the light delivered to the treated area. It improves the homogenization of the light dose by adapting the light delivery to untreated or not completely treated regions. The second benefit comes from the fact that visual assessment of the dosimetry is more natural than the punctual evaluation of discrete measurements. Moreover, the use of a gradient dose display allows a quick appreciation of the dose being delivered. Ultimately, one can obtain images as those used in radiotherapy treatment planning (Figure 8).

The last benefit regards the procedure time. The proposed approach reduces the intervention time by avoiding the setting of the measurements probes inside the thorax which take about 15 minutes in te case of intrapleural PDT for MPM. 


\section{Conflict of interest}

All the authors have no conflict of interest to declare. 


\section{References}

[1] Beyer W. Systems for light application and dosimetry in photodynamic therapy. Journal of Photochemistry and Photobiology B: Biology 36 :153-156 1996

[2] Mordon S, Cochrane C Tylcz JB Betrouni N Mortier L Koncar V. Light emitting fabric technologies for photodynamic therapy. Photodiagnosis Photodyn Ther. 12 (1):1-8 2015

[3] Lianga X, Kundua P Finlay J Goodwin M Zhu T. Maximizing fluence rate and field uniformity of light blanket for intraoperative PDT. Proc SPIE Int Soc Opt Eng.2012 January $21 ; 8210$ : .doi:10.1117/12.908493.2012

[4] Zhu TC, Kim MM Liang X Lio B Meo JL Finlay JC Dimofte A Rodriguez C Simone CB Cengel K Friedberg JS. Real-time treatment feedback guidance of pleural PDT. Proc SPIE Int Soc Opt Eng. 8568 1-11 2013

[5] H. B. Ris, H. J. Altermatt, R. Inderbitzi, R. Hess, B. Nachbur, J. C. Stewart, Q. Wang, C. K. Lim, R. Bonnett, M. C. Berenbaum. Photodynamic therapy with chlorins for diffuse malignant mesothelioma: initial clinical results. Br J Cancer 64 (1116):1120-1991

[6] Friedberg JS, Mick R, Stevenson J, Metz J, Zhu T, Buyske J, Sterman DH, Pass HI, Glatstein E, Hahn SM. A phase I study of Foscan-mediated photodynamic therapy and surgery in patients with mesothelioma. Ann Thorac Surg. 2003 Mar;75(3):952-9.

[7] Friedberg JS, Mick R Culligan M. Photodynamic Therapy and the Evolution of a LungSparing Surgical Treatment for Mesothelioma. Ann Thorac Surg. 91 :1738-1745 2011

[8] Friedberg JS, Culligan MJ, Mick R, Stevenson J, Hahn SM, Sterman D, Punekar S, Glatstein E, Cengel K. Radical pleurectomy and intraoperative photodynamic therapy for malignant pleural mesothelioma. Ann Thorac Surg. 2012 93(5):1658-65.

[9] Dimofte A, Zhu TC Jarod C. Finlay. In vivo light dosimetry for pleural PDT. 2009, 71640A In: Kessel DH, editor:1-12 2009.

[10] Zhu TC. Dosimetry in pleural photodynamic therapy. J Natl Compr Canc Netw 2012;10:S-60. $10: 60-2012$.

[11] Friedberg JS, Mick R, Stevenson JP, Zhu T, Busch TM, Shin D, Smith D, Culligan, M, Dimofte A, Glatstein E, Hahn SM. Phase II trial of pleural photodynamic therapy and surgery for patients with non-small-cell lung cancer with pleural spread. J Clin Oncol. 2004 Jun $1 ; 22(11): 2192-201$.

[12] Munck C., Mordon S., Betrouni N. Illumination profile characterization of a light device for the dosimetry of intra-pleural photodynamic therapy for mesothelioma. Photodiagnosis and Photodynamic Therapy, under press, 2016.

[13] Betrouni N, Colin P Puech P Villers A Mordon S. An image guided treatment platform for prostate cancer photodynamic therapy. Conf Proc IEEE Eng Med Biol Soc 370-373 2013 Osaka, Japan

[14] Stepp H, Beck T Pongratz T Meinel T Kreth FW Tonn JCh Stummer W. ALA and malignant glioma: fluorescence-guided resection and photodynamic treatment. $\mathrm{J}$ Environ Pathol Toxicol Oncol. 26 (2):157-164 2007

[15] Karakullukcu B., van Veen R. Aans J. Hamming-Vrieze O Navran A. Teertstra J van den Boom F. Niatsetski Y. Sterenborg H. Tan B. MR and CT Based Treatment Planning formTHPCMediated Interstitial Photodynamic Therapy of Head and Neck Cancer: Description of the Method. Lasers in Surgery and Medicine 45 :517-523 2013

[16] Oakley E., Wrazen B. Bellnier D. Syed Y. Arshad H. Shafirstein G. A New Finite Element Approach for Near Real-Time Simulation of Light Propagation in Locally Advanced Head and Neck Tumors. Lasers in Surgery and Medicine 47 :60-67 2015 
[17] S.R.Mordon, M. E. Vuylsteke P. Mahieu N. Betrouni. Endovenous laser treatment of the great saphenous vein: Measurement of the pullback speed of the fiber by magnetic tracking. IRBM 34 (3):252-256 2013

[18] Sadick N., Rochon P. Mordon S. Advantages of real-time magnetic tracking of the cannula for controlled laser assisted lipolysis. 2010 ASLMS Annual Conference, Phoenix, AZ, USA, April 16-18, 2010Lasers in Surgery and Medicine, 2010, Supp 22, 65: 1962010. 


\section{Figures captions}

Figure 1: The light applicator. (a) The position of the 3D sensor according to the cylindrical diffuser. (b) Assembly of the applicator.

Figure 2:. Illumination profile of the applicator when connected to laser emitting $3 \mathrm{~W}$.

Figure 3: (Left) Thoracic phantom with surgical opening and isotropic probes placement in the pleural wall. (Right) Photodynamic therapy procedure simulation.

Figure 4: 3D Computerized Tomography of the thoracic phantom with patterns created by the fiducial markers. Images are displayed using three orientations: transversal, sagittal and coronal.

Figure 5: 3D light dose display combined with CT images of the thorax cavity phantom. Crossing lines (top left viewer (sagittal), top right viewer (coronal) and center right viewer (axial)) indicate the light applicator position inside the cavity.

Figure 6: Cumulated fluence measured in one unique position over time by the two methods: our proposed model based method (Model_Measurments) and the reference method based on a probe and a powermeter (Ref_Measurments). Six independent experiments were done. (a) Measurements: Exp 1, Exp 2 and Exp 5. (b) Measurements: Exp 4, Exp 3 and $\operatorname{Exp} 6$.

Figure 7: Temporal evolution of the light dose (fluence $\mathrm{J.cm}^{-2}$ ) measured in one position by the two methods: Reference method based on a dosimeter (power-meter) and Model method assuming a unique profile emission from the light applicator after correction.

Figure 8: Example of a treatment planning display for a lung radiotherapy showing the distribution of radiation doses delivered to the tumor. The prescription dose is 50 gray. 

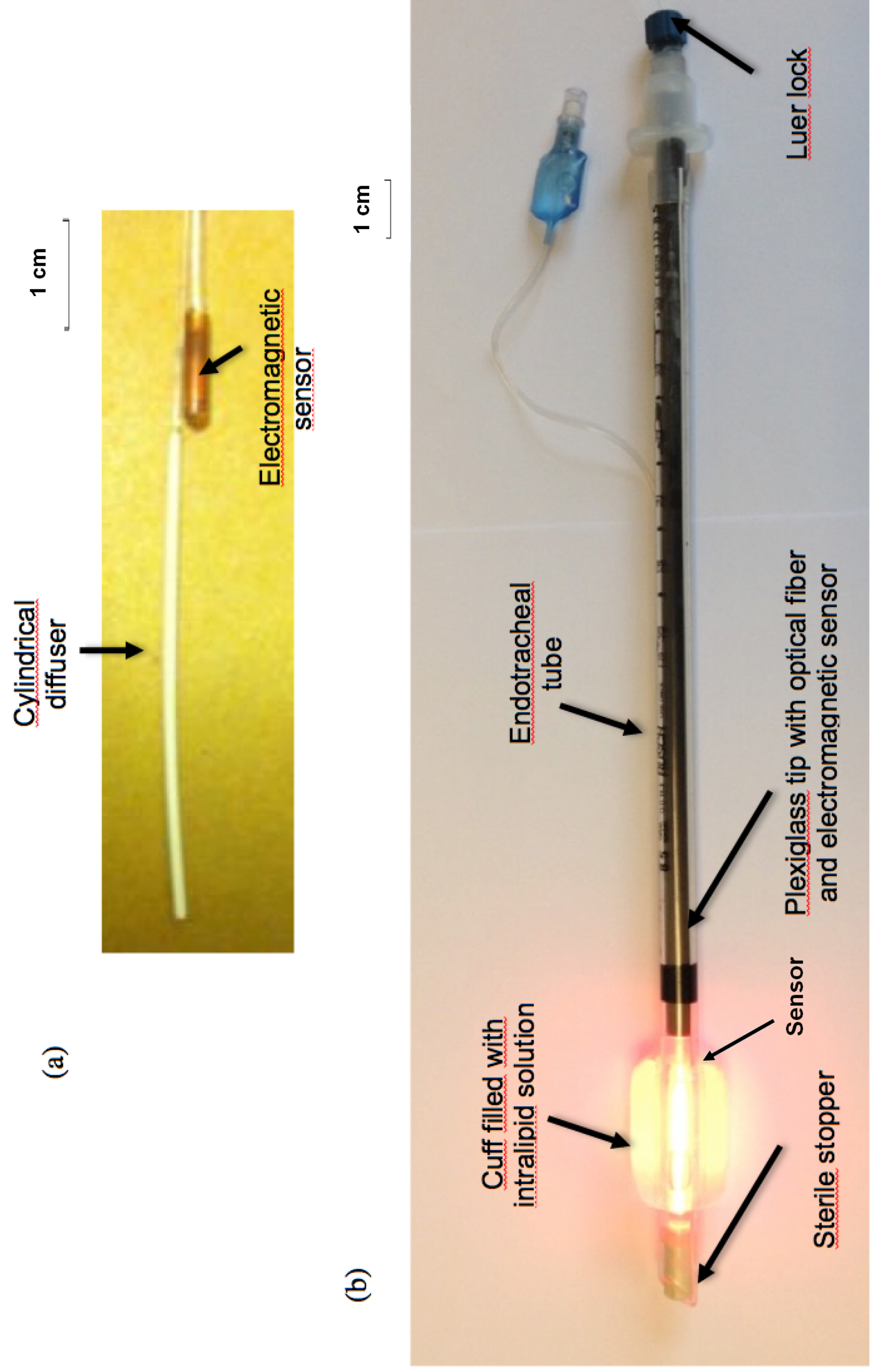


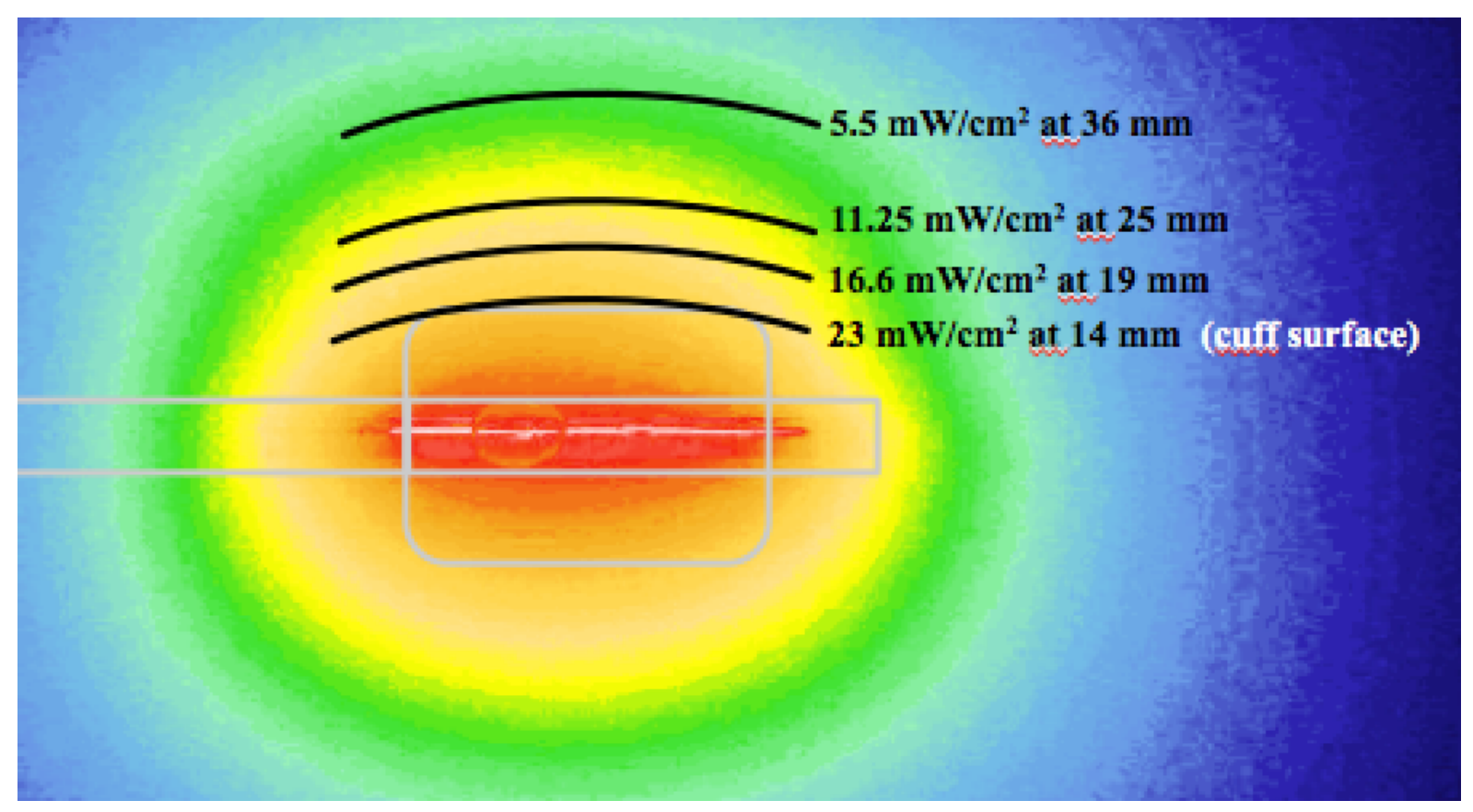



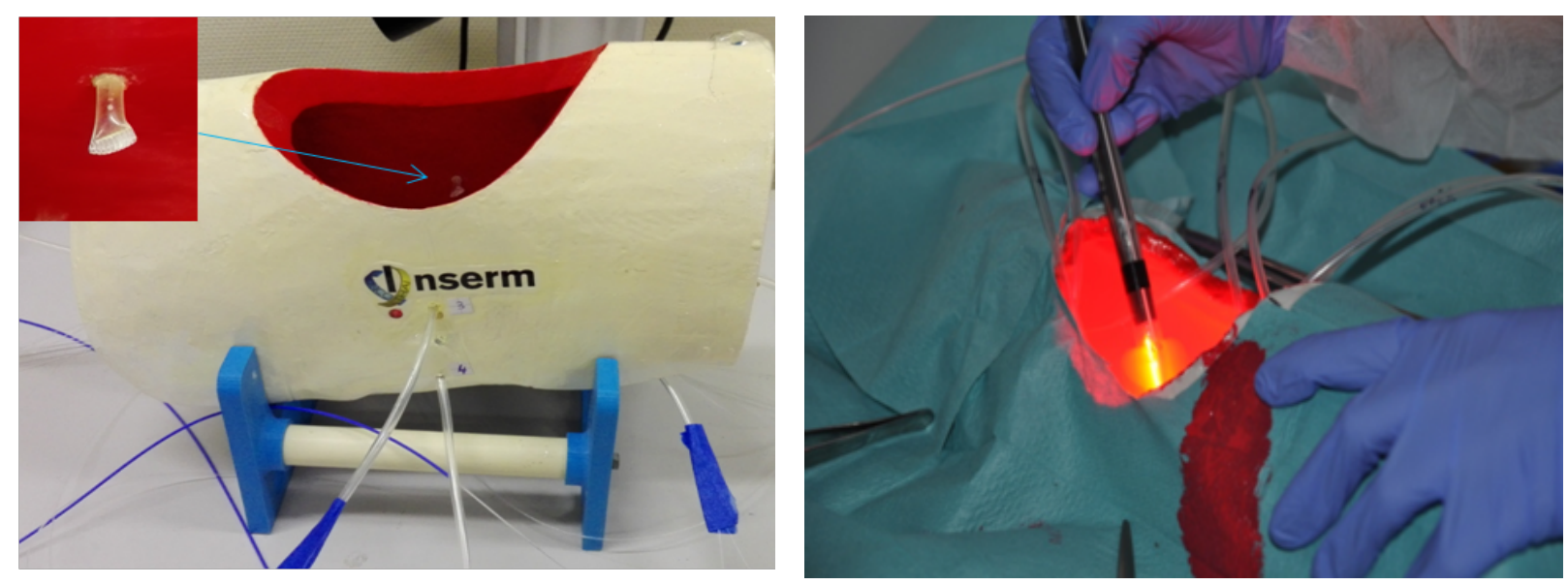


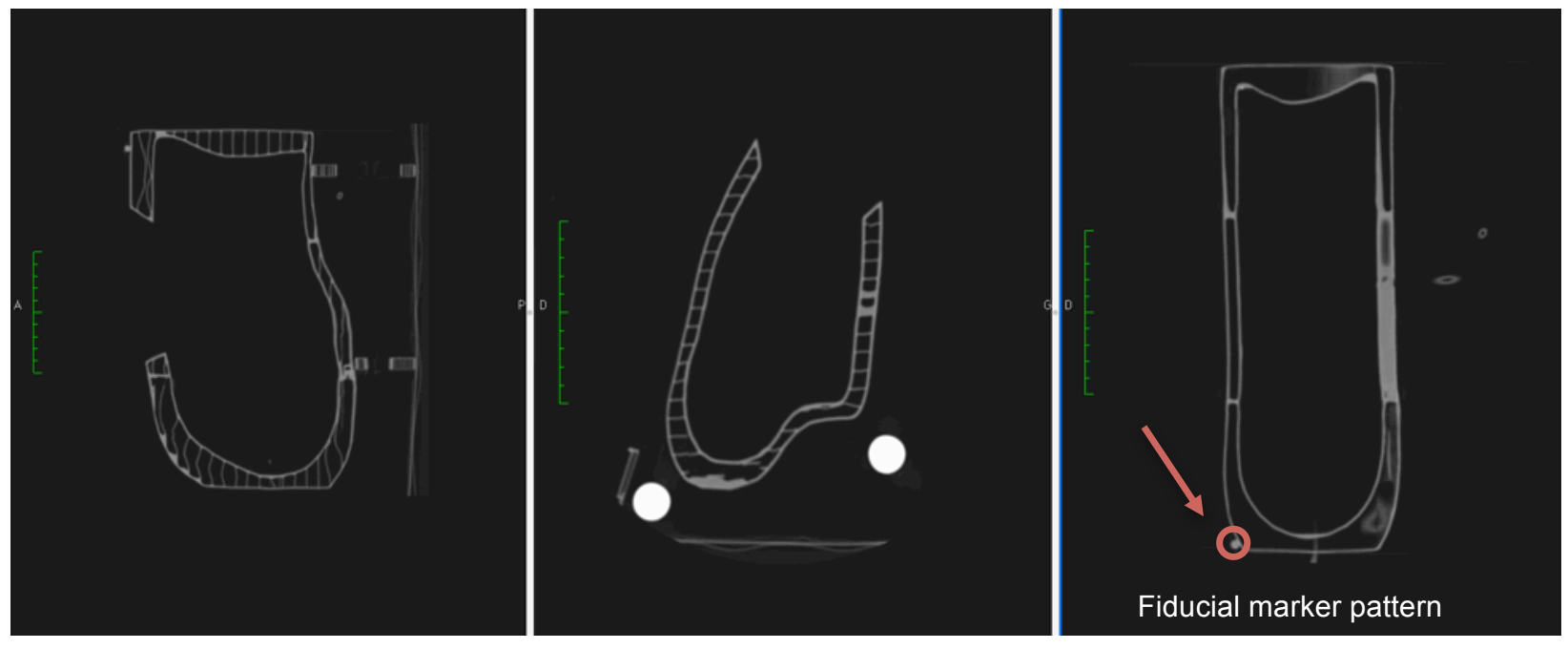




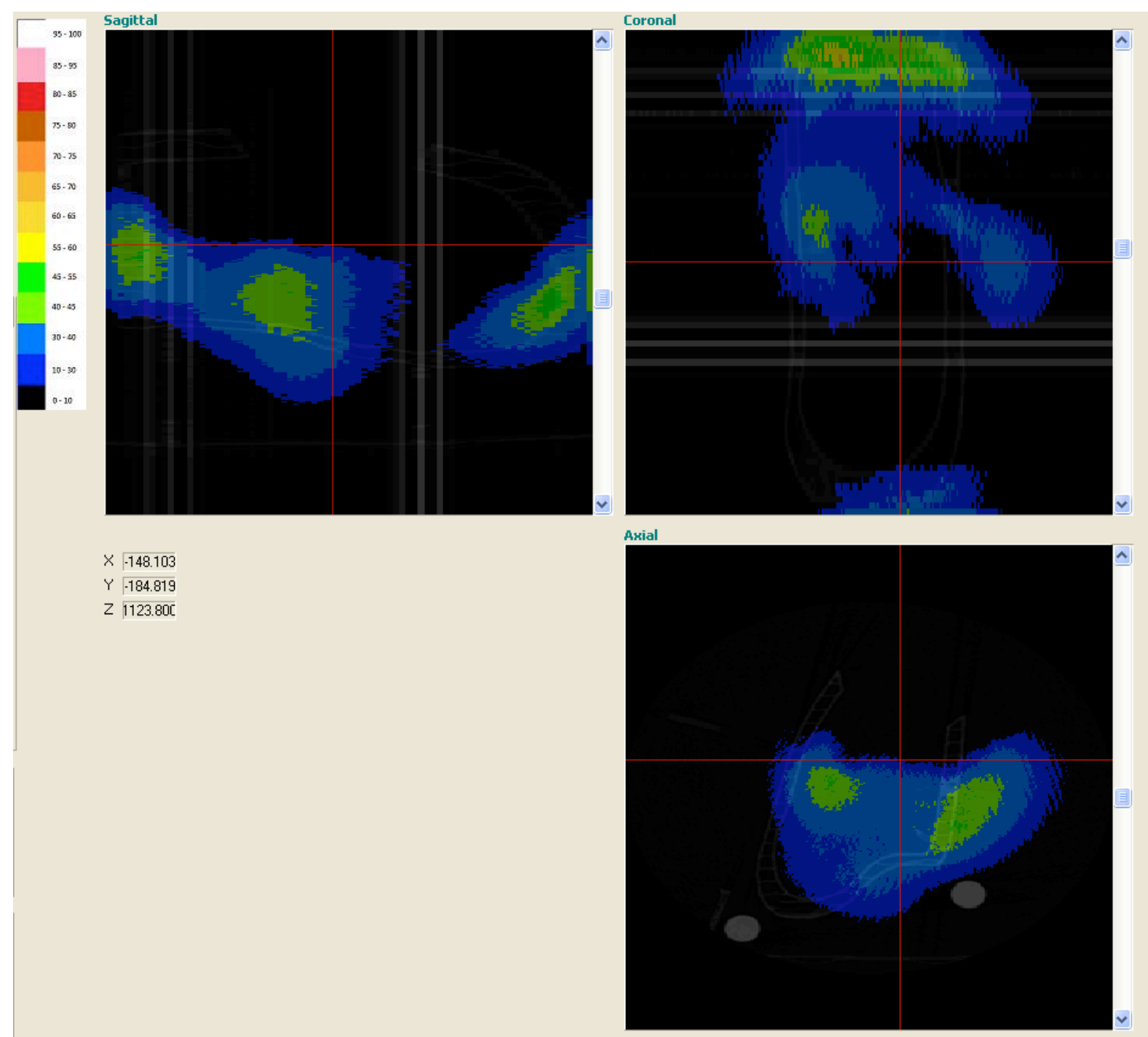




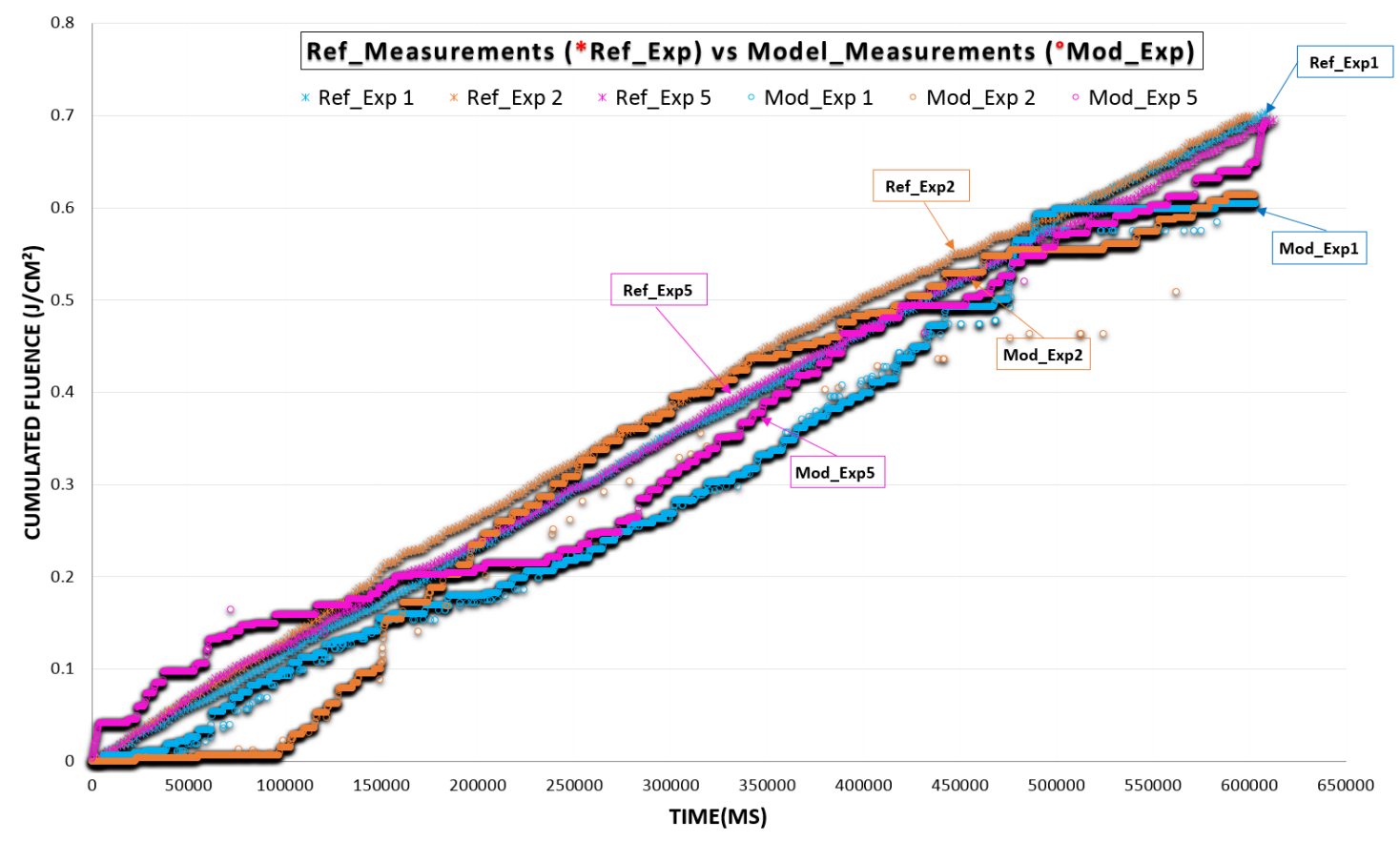

(a)

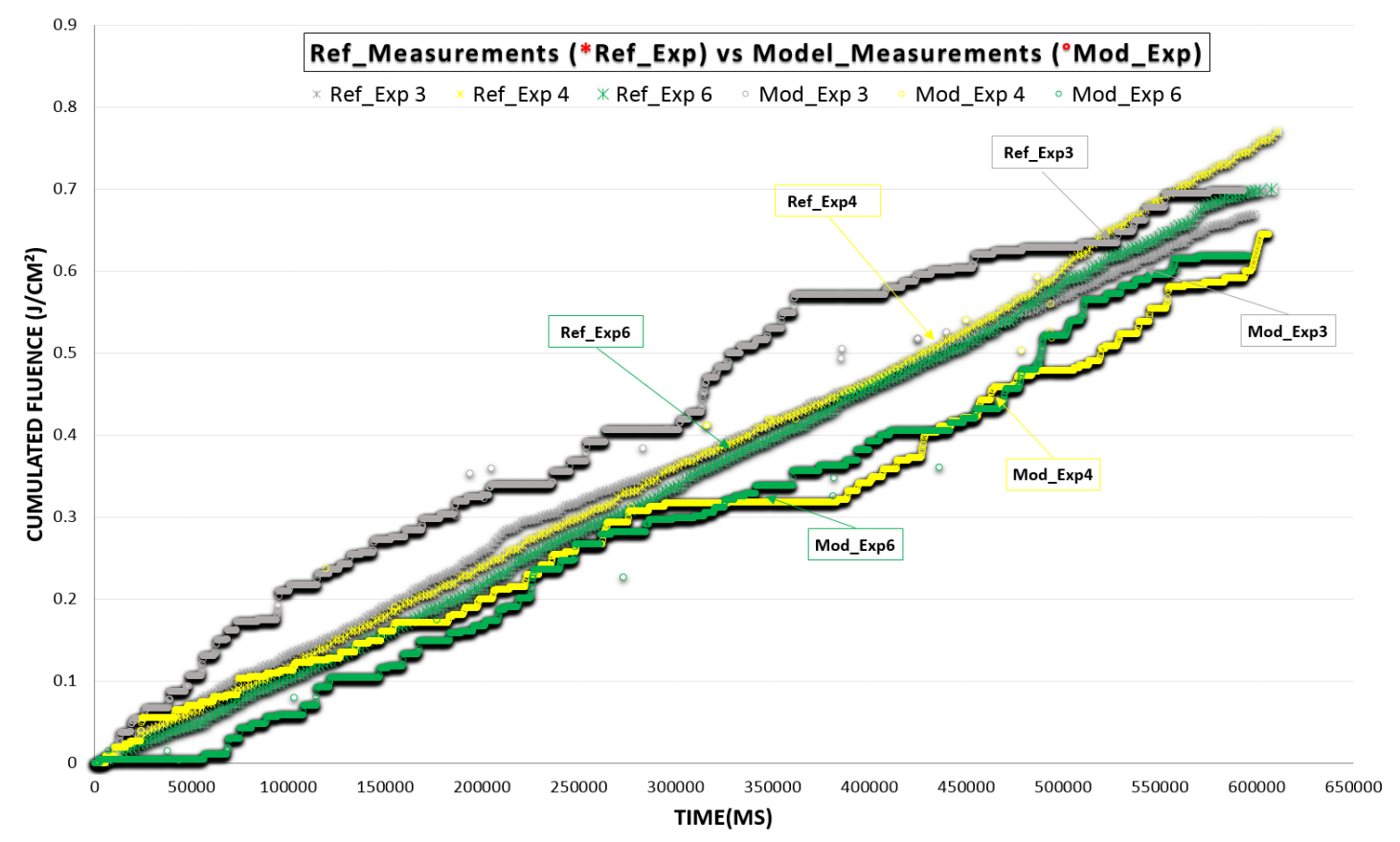

(b) 


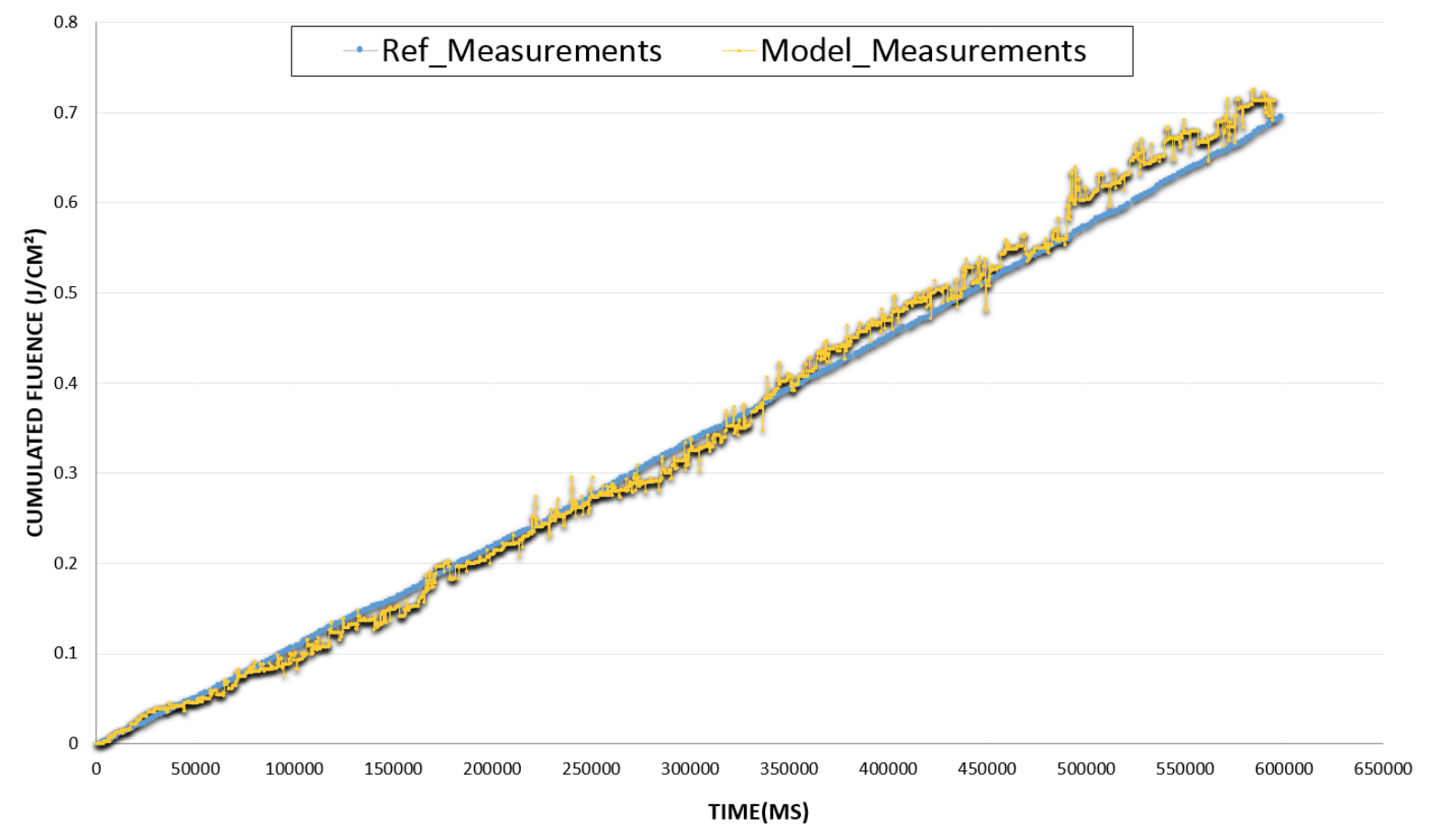




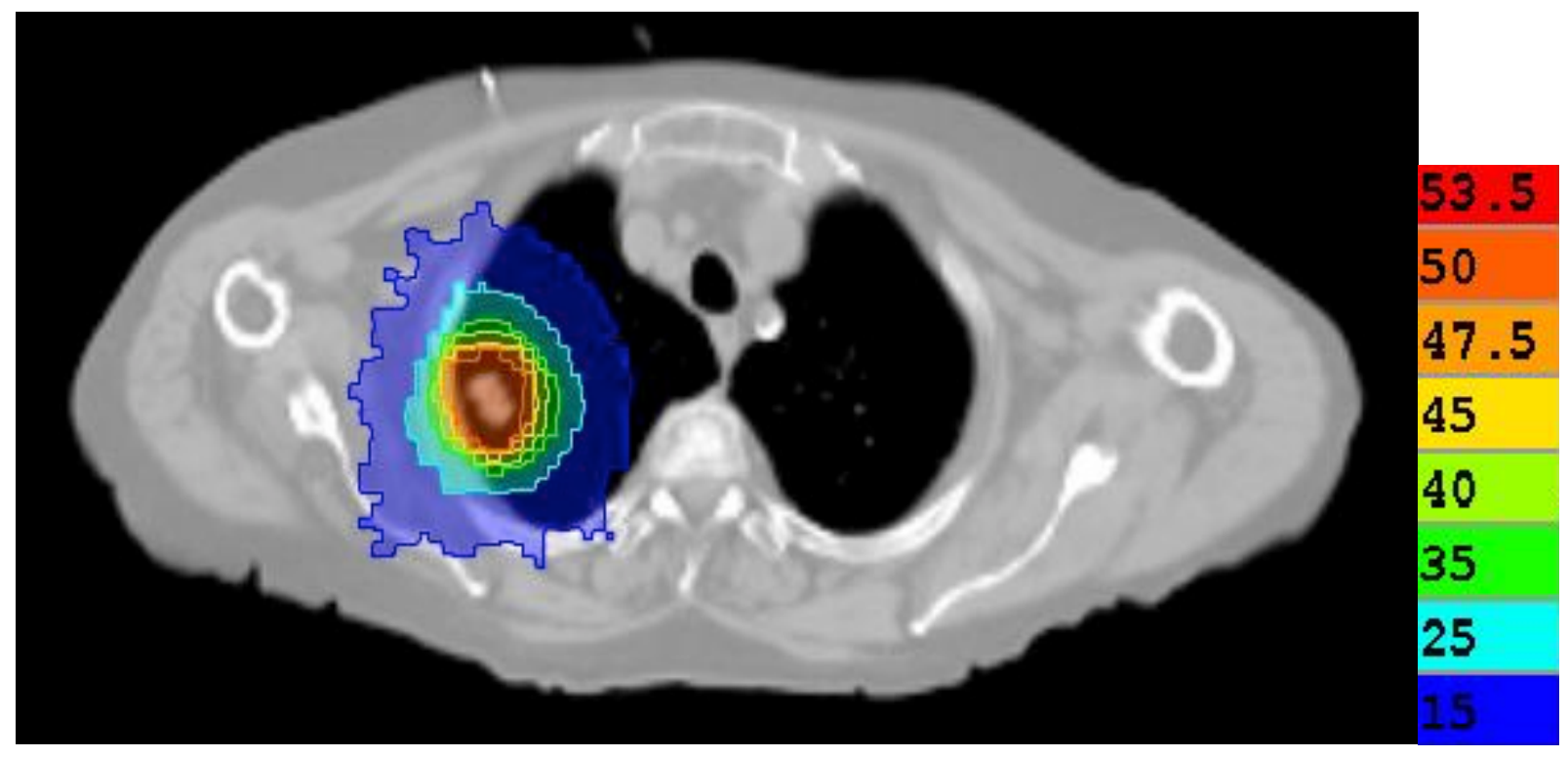

